\title{
Conhecimento das mulheres acerca do exame preventivo do câncer de colo de útero
}

\author{
Women's knowledge about the preventive examination for cervical cancer \\ Conocimientos de las mujeres sobre el examen preventivo del cáncer de cuello uterino
}

Recebido: 20/11/2021 | Revisado: 28/11/2021 | Aceito: 29/11/2021 | Publicado: 30/11/2021

\author{
Meury Amorim Rezende \\ ORCID: https://orcid.org/0000-0001-7526-7023 \\ Faculdade Guaraí, Brasil \\ E-mail: meuryrezende@gmail.com \\ Gessilene de Araújo Santos Oliveira \\ ORCID: https://orcid.org/0000-0003-3004-2714 \\ Faculdade Guaraí, Brasil \\ E-mail: gessilene_10 @ hotmail.com \\ Glaucya Wanderley Santos Markus \\ ORCID: https://orcid.org/0000-0001-8916-1086 \\ Faculdade Guaraí, Brasil \\ E-mail: glaucyamarkus@outlook.com \\ Reobbe Aguiar Pereira \\ ORCID: https://orcid.org/0000-0003-2578-2611 \\ Universidade Brasil, Brasil \\ E-mail: enfreobbe@gmail.com \\ Giullia Bianca Ferraciolli do Couto \\ ORCID: https://orcid.org/0000-0002-9768-778X \\ Faculdade Guaraí, Brasil \\ E-mail: giulliabianca@hotmail.com \\ Adriana Keila Dias \\ ORCID: https://orcid.org/0000-0003-1291-5593 \\ Faculdade Guaraí, Brasil \\ E-mail: adrianakeiladias@hotmail.com \\ Camilla Teles Alencar \\ ORCID: https://orcid.org/0000-0002-5767-9267 \\ Faculdade Guaraí, Brasil \\ E-mail: camillatalencar@gmail.com \\ Karla Camila Correia da Silva \\ ORCID: https://orcid.org/0000-0003-1538-7028 \\ Faculdade Guaraí, Brasil \\ E-mail:karlacamilac@yahoo.com.br
}

\begin{abstract}
Resumo
O câncer do colo do útero consiste em um problema de saúde pública devido sua alta incidência no país, nesse contexto encontra-se o exame preventivo do câncer do colo do útero (PCCU), que é fundamental para o diagnóstico precoce da doença. Assim, este estudo tem como objetivo em identificar o conhecimento das mulheres do município do interior do Tocantins, acerca da importância da realização do exame Preventivo do Câncer de Colo de Útero. Trata-se de uma pesquisa de campo, transversal, com análise qualiquantitativa, realizada com mulheres atendidas pela Clínica de Educação Para Saúde (CEPS) do Instituto Educacional Santa Catarina - Faculdade Guaraí (IESC-FAG), em Guaraí - Tocantins. Participaram da pesquisa 30 mulheres de forma voluntária com idades entre 25 e 64 anos, que realizaram consultas na Clínica Escola do Instituto Educacional Santa Catarina - Faculdade Guaraí (IESC-FAG). O estudo aponta que as participantes possuem conhecimento regular acerca do exame de PCCU onde 100\% (30) reconhecem e compreendem a importância da realização do exame e 96,7 (29) sua finalidade. Dessa maneira a enfermagem deve atentar-se cada vez mais para a oportunidade de esclarecimentos de dúvidas no momento da consulta, propiciando uma escuta de excelência que além da realização técnica do exame engloba todos os seus aspectos, levando ainda mais conhecimento para as mulheres, visto que este é um fator fundamental para a adesão ao exame, sendo possível assim reduzir a incidência da patologia em estudo.
\end{abstract}

Palavras-chave: Exame citopatológico; Prevenção; Promoção à saúde; Saúde da mulher.

\section{Abstract}

Cervical cancer is a public health problem due to its high incidence in the country, in this context there is the preventive examination for cervical cancer (PCCU), which is essential for the early diagnosis of the disease. Thus, this study aims to identify the knowledge of women in the countryside of Tocantins, about the importance of performing the preventive examination for cervical cancer. This is a cross-sectional field research, with qualitative and quantitative analysis, carried out 
with women assisted by the Health Education Clinic (CEPS) of the Santa Catarina Educational Institute - Faculdade Guaraí (IESC-FAG), in Guaraí - Tocantins. The study points out that the participants have regular knowledge about the PCCU exam where $100 \%$ (30) recognize and understand the importance of taking the exam and 96.7 (29) its purpose. Thus, nursing must pay increasing attention to the opportunity to clarify doubts at the time of consultation, providing an excellent listening that, in addition to the technical performance of the examination, encompasses all its aspects, bringing even more knowledge to women, as that this is a fundamental factor for adherence to the exam, thus being possible to reduce the incidence of the studied pathology.

Keywords: Cytopathological exam; Prevention; Health promotion; Women's health.

\section{Resumen}

El cáncer de cuello uterino es un problema de salud pública debido a su alta incidencia en el país, en este contexto se encuentra el examen preventivo del cáncer de cuello uterino (UPC), que es fundamental para el diagnóstico precoz de la enfermedad. Así, este estudio tiene como objetivo identificar el conocimiento de las mujeres del campo de Tocantins, sobre la importancia de realizar el examen preventivo del cáncer de cuello uterino. Se trata de una investigación de campo transversal, con análisis cualitativo y cuantitativo, realizada con mujeres atendidas por la Clínica de Educación para la Salud (CEPS) del Instituto Educativo Santa Catarina - Faculdade Guaraí (IESC-FAG), en Guaraí - Tocantins. Treinta mujeres, de entre 25 y 64 años, que participaron en la investigación, participaron en la investigación de manera voluntaria en la Clínica Escolar del Instituto Educativo Santa Catarina - Faculdade Guaraí (IESC-FAG). El estudio señala que los participantes tienen un conocimiento regular sobre el examen PCCU donde el 100\% (30) reconocen y comprenden la importancia de tomar el examen y 96,7 (29) su propósito. Así, la enfermería debe prestar cada vez más atención a la oportunidad de aclarar dudas en el momento de la consulta, brindando una excelente escucha que, además de la ejecución técnica del examen, engloba todos sus aspectos, aportando aún más conocimientos a las mujeres, ya que este es un factor fundamental para la adherencia al examen, pudiendo así reducir la incidencia de la patología estudiada.

Palabras clave: Examen citopatológico; Prevención; Promoción de la salud; Salud de la mujer.

\section{Introdução}

O câncer é uma das principais causas de morte em todo o mundo, mesmo que muitos dos tipos de canceres possam ser identificados e tratados no início, com algumas formas de prevenção. Um dos tipos que têm alta incidência é o câncer de colo de útero, onde estima-se que para cada ano de 2020/2022, sejam diagnosticados 16.590 novos casos do câncer no Brasil. No entanto, o mesmo deixou de ser tão letal com a realização do exame preventivo de câncer de colo de útero (PCCU), que ajuda a identificar de forma precoce o que pode vir a se tornar um câncer (Amaral; Gonçalves; Silveira, 2017 \& Iarc, 2020).

Apesar da prevenção ser de fácil acesso de ser realizado e de ter um prognostico considerado bom, o câncer de colo de útero, ainda está entre um dos principais tipos de canceres que ocorre nas mulheres, onde no ano de 2019 ocorreram 6.596 óbitos devido ao câncer do colo de útero representando uma taxa de 5.33/100 mil mulheres. Isso mostra que mesmo com tudo que se sabe sobre o mesmo, ele ainda leva ao óbito em altas taxas, por ser tratar de um tipo que as vezes começa silencioso (Brasil, 2021; Martins \& Motta, 2020).

As políticas públicas brasileiras, acerca da saúde da mulher, garantem que as mulheres tenham acesso aos serviços de saúde, como o exame preventivo do câncer de colo de útero. Mas o que se pode observar, em meio a grande incidência do câncer de colo de útero, sendo a terceira localização primária de incidência e a quarta de mortalidade por câncer em mulheres no país, é que apesar das políticas públicas resguardarem o acesso das mulheres a esses cuidados, nem todos os lugares estão prontos e tem a disponibilidade desses serviços (Brasil, 2019 \& Fernandes, et al., 2019).

É possível verificar entre as mulheres uma certa dificuldade em realizar o exame, por falta de conhecimento, de como o procedimento funciona. Também tem os estigmas acerca desse procedimento, como algumas mulheres relatam as outras que sentiram alguma dor durante a realização do exame, algumas mulheres também sentam vergonha, por estarem se expondo de certa forma a outras pessoas. Outro fator que desestimula as mulheres a não realizar esse exame, e a falta de acesso ao mesmo, tendo em vista, que mesmo que as políticas públicas garantam esse acesso, nem todos os órgãos públicos fornecem esse serviço (Melo, et al., 2019). 
Tendo em vista que muitos municípios passam por dificuldades, quanto a prestação desse serviço, pode ser visto que muitos municípios, passam por períodos em que não realizam essa coleta, as vezes por falta de matéria, ou até mesmo, a falta de ter um laboratório, para o qual possam mandar o material colhido nos exames (Teixeira, et al., 2018).

Observa-se também que muitas mulheres não têm o conhecimento de que o exame preventivo de câncer de colo de útero, pode ser realizado pelo Sistema Único de Saúde. Observa-se também, que as mulheres de menor condição financeira, tem ainda menos acesso a informação, uma vez que só procuram as unidades básicas de saúde, quando já apresentam sintomas, muitas vezes exacerbados. Existe uma dificuldade dessas mulheres ao acesso à informação, sobre o que realmente é o câncer de colo de útero, o que causa essa enfermidade, quais os sinais e sintomas que apresentam, e a sua forma de prevenção (Silva, 2019).

A informação acerca dessa prevenção e algo que está a cargo das unidades básicas de saúde, como parte das políticas públicas, que são utilizadas para prevenir o câncer de colo de útero (Gasparin, et al., 2020).

Dentro das políticas públicas, o Ministério da Saúde preconiza que os profissionais de enfermagem, atuem na prevenção e na informação a respeito do câncer de colo uterino, através da consulta de enfermagem. Dentro da consulta de enfermagem, e possível desenvolver um relacionamento terapêutico com a paciente, o enfermeiro promove o acolhimento e uma boa escuta para promoção da saúde, promovendo um ambiente confortável, trazendo a confiança do paciente acerca da prevenção (Carvalho, et al., 2016).

Para que mulher se sinta segura na realização de um exame que é invasivo, e acaba por expor a paciente, o enfermeiro desempenha um papel muito importante para o sucesso dessa coleta. Pois, o enfermeiro é o profissional que fica à frente da coleta, e este profissional tem o dever de passar confiança para a paciente, de forma que a mesma não se sinta constrangida em momento algum, e consiga conversar com o profissional sobre todas as queixas que a levaram a estar procurando esse serviço, ou se ela apenas resolveu fazer o exame como rotina de um acompanhamento anual (Gasparin, et al., 2020).

Alguns fatores influenciam de forma significativa o surgimento do câncer de colo de útero, como infecção pelo papiloma vírus humano, especificamente os subtipos HPV-16 e o HPV-18, que geralmente são culpados por 70\% dos tipos de canceres cervicais. Temos outros fatores de risco, como o tabagismo, a iniciação da vida sexual precoce, ao grande número de parceiros sexuais, assim como o uso de contraceptivos orais, é considerado fatores de risco para a predisposição ao desenvolvimento do câncer de colo de útero (Melo, et al., 2019).

Assim observa-se que além das medidas de prevenção, algo importante deve ser feito, que é o esclarecimento das mulheres a respeito do que é o câncer de colo de útero, e a importância de todas as medidas preventivas (Acosta, et al., 2017).

Diante de todos esses dados, levantou-se o seguinte questionamento: Qual o conhecimento que as mulheres têm acerca do que é o exame preventivo de câncer de colo de útero?

Assim o presente trabalho tem o objetivo de identificar o conhecimento das mulheres do município do interior do Tocantins, acerca da importância da realização do exame Preventivo do Câncer de Colo de Útero. Proporcionando assim a identificação de que tipo de ajuda pode ser fornecida a essas mulheres para que as mesmas entendam a importância do procedimento, e possam realiza-lo.

\section{Material e Métodos}

O presente estudo trata-se de uma pesquisa de campo, transversal, com análise qualiquantitativa, realizada com mulheres atendidas pela Clínica de Educação Para Saúde (CEPS) do Instituto Educacional Santa Catarina - Faculdade Guaraí 
(IESC-FAG), em Guaraí - Tocantins. Fizeram parte da pesquisa 30 mulheres que foram atendidas na Clínica, entre os meses de janeiro a outubro de 2021, com idades entre 25 a 64 anos.

Segundo Pereira et al., (2018), os trabalhos que contemplam análise qualiquantitativa possuem em geral uma abordagem quantitativa alcançada por meio de diferentes instrumentos como entrevistas e questionários que iram proporcionar as pesquisas dos numéricos que posteriormente serão complementados por dados qualitativos.

O estudo foi realizado obedecendo aos princípios éticos da pesquisa com seres humanos, preconizados na Resolução nº. 466/2012 do Conselho Nacional de Saúde (Brasil, 2012), sendo submetido à apreciação do Comitê de Ética em Pesquisa da Fundação Escola de Saúde Pública de Palmas, campus Palmas-TO e aprovado sob o parecer nº 5.082.464.

Em decorrência da pandemia causada pela Covid-19, a coleta de dados deu-se pela busca do contato telefônico no cadastro das mulheres assistidas na Clínica de Educação Para Saúde CEPS da instituição IESC/FAG, campos III, no município de Guaraí-TO, onde de maneira particular via WhatsApp ou ligação telefônica, estas mulheres receberam o envio de um link dando acesso ao Termo de Consentimento Livre e Esclarecido (TCLE) por meio do Google Forms, mantendo assim as recomendações do Ministério da Saúde acerca do distanciamento social.

Utilizando o mesmo link, as participantes tiveram acesso ao questionário virtual semiestruturado, elaborado pelas próprias autoras do estudo, contendo 15 perguntas, sendo que destas 3 foram destinadas a traçar o perfil socioeconômico das participantes e 12 ligadas diretamente ao conhecimento acerca do exame de PCCU com 8 questões fechadas e 4 abertas.

\section{Resultados e Discussão}

Participaram da pesquisa 30 mulheres de forma voluntária com idades entre 25 e 64 anos, que realizaram consultas na CEPS do Instituto Educacional Santa Catarina - Faculdade Guaraí (IESC-FAG).

A Clínica de Educação Para a Saúde, propõe atender a população que necessita de assistência à saúde, em relação a atenção do programa saúde da mulher que visa assistência qualificada em cuidados, e prevenção do câncer do colo do útero, as consultas de enfermagem com finalidade de assegurar a mulher na linha de rastreamento a lesões precursoras do câncer uterino, ao diagnóstico precoce, e terapia adequada.

No período de estudo que compreendeu entre os meses de janeiro a outubro de 2021, a Clínica possui capacidade de atender uma média de 240 mulheres, tendo em vista que recebe em média 3 pacientes ao dia e oferece seus atendimentos duas vezes por semana, no entanto, na realidade este número torna-se inferior devido ao baixo número de agendamentos, tendo como um dos motivos o medo das mulheres em buscar atendimento em meio ao período pandêmico. 
Tabela 1: Perfil socioeconômico das participantes.

\section{PERFIL NÚMERO TOTAL PORCENTAGEM}

\section{FAIXA ETÁRIA}

\begin{tabular}{|c|c|c|}
\hline 25 a 30 anos & 17 & $56,7 \%$ \\
\hline 31 a 40 anos & 7 & $23,3 \%$ \\
\hline 41 a 50 anos & 4 & $13,3 \%$ \\
\hline 51 a 64 anos & 2 & $6,7 \%$ \\
\hline \multicolumn{3}{|l|}{ ESCOLARIDADE } \\
\hline Ensino fundamental incompleto & 1 & $3,3 \%$ \\
\hline Ensino fundamental completo & 1 & $3,3 \%$ \\
\hline Ensino médio completo & 5 & $16,7 \%$ \\
\hline Ensino superior incompleto & 18 & $60 \%$ \\
\hline Ensino superior completo & 5 & $16,7 \%$ \\
\hline \multicolumn{3}{|l|}{ RENDA FAMILIAR } \\
\hline Até 1 salário mínimo & 12 & $40 \%$ \\
\hline 1 a 2 salários mínimos & 12 & $40 \%$ \\
\hline 2 a 3 salários mínimos & 6 & $20 \%$ \\
\hline
\end{tabular}

Fonte: Autores (2021).

A Tabela 1, demonstra o perfil socioeconômico das participantes, onde pode-se perceber que a maioria da amostra foi constituída por mulheres na faixa etária de 25 a 30 anos seguido de 31 a 40 anos de idade. Em relação à escolaridade verificouse maior número com ensino superior incompleto chegando a compor 60\% (18) da amostra, conseguinte aparece o ensino superior completo e ensino médio completo que alcançaram percentuais iguais $(16,7 \%)$. A tabela, também refere-se a renda familiar, onde $40 \%$ (12) das participantes afirmaram ter de 1 a 2 salários mínimos, se igualando ao número das que referiram receber até um salário mínimo, enquanto $20 \%$ das participantes declararam renda entre 2 a 3 salários mínimos.

Estes dados diferem de outros estudos que apontaram como maior parte da amostra mulheres acima dos 31 anos de idade (Dantas, et al., 2018 \& Vasconcelos, et al., 2017). O estudo de Melo, et al., (2019), verificou-se uma prevalência de mulheres na faixa etária entre 40 e 59 anos, possuindo ensino fundamental completo e renda familiar de até um salário mínimo.

Segundo Silva, et al., (2021), tanto a baixa escolaridade quanto a baixa renda familiar estão associadas a não realização do exame de PCCU.

Essa afirmação se dá pelo fato de que mulheres com renda baixa, bem como com pouca instrução escolar costumam ter também conhecimento insuficiente acerca do processo de saúde negligenciando os cuidados com a mesma, deixando de realizar o exame preventivo em função da falta de compreensão acerca de sua finalidade e importância. 
Gráfico 1: Importância e acesso ao exame de PCCU.

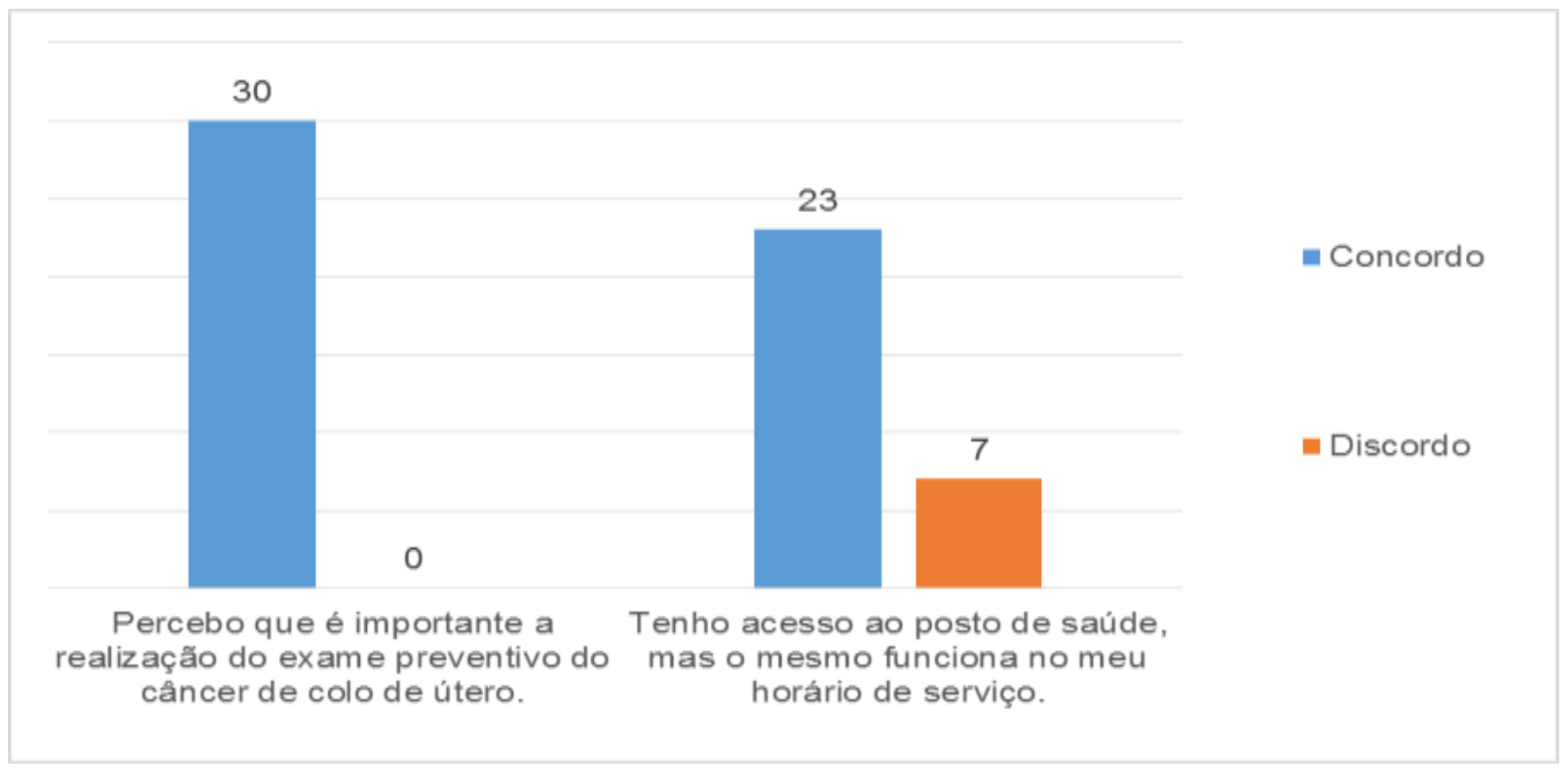

Fonte: Autores (2021).

A partir do Gráfico 1, observa-se que 100\% (30) das mulheres da amostra percebem que é importante a realização do exame preventivo do câncer de colo de útero, no entanto, 76,6\% (23) dessas afirmam que tem acesso ao posto de saúde, porém este funciona em seu horário de serviço.

Os dados acima evidenciam um lado bastante positivo onde demonstra que todas as mulheres reconhecem a importância do exame preventivo sendo este um fator importante para sua decisão por outro lado é preocupante o fato de que os serviços de saúde possuem horários semelhantes ao de seu trabalho.

Para Silva, et al., (2021), um dos fatores que contribui para não realização do exame está na disponibilidade dos serviços.

Essa disponibilidade vai muito além do pressuposto de oferecer o exame, onde os serviços de saúde devem estar atentos para as reais necessidades das mulheres, podendo ser oferecido o exame em horários alternativos a fim de melhor atender suas usuárias aumentando assim a adesão ao exame. 
Gráfico 2: Vergonha e o uso de contraceptivo em relação ao exame de PCCU.

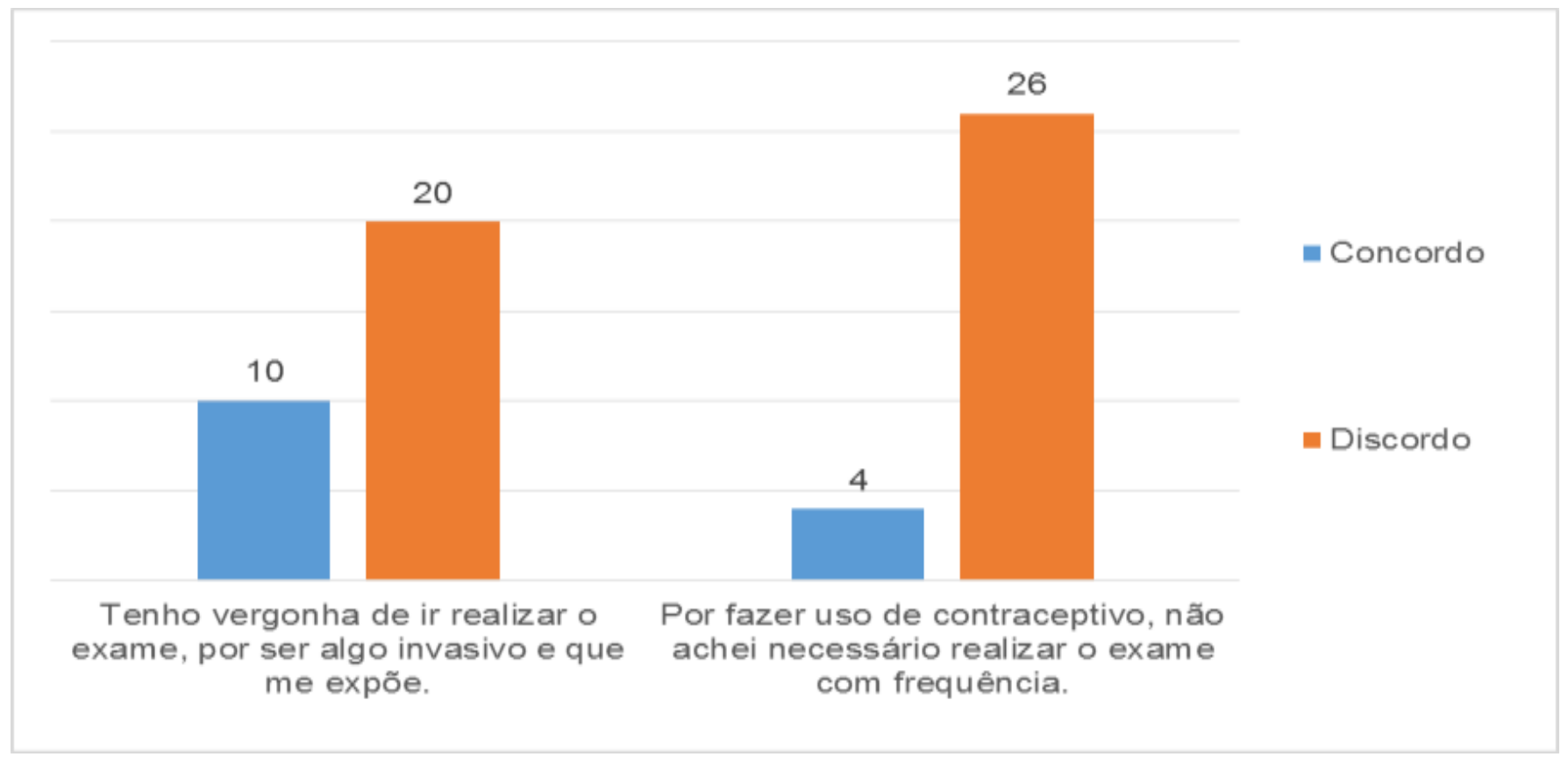

Fonte: Autores (2021).

De acordo com o gráfico supracitado, 33,4\% (10) das mulheres afirmam que têm vergonha de realizar o exame preventivo do colo do útero por se tratar de algo invasivo e acaba expondo às mulheres, no entanto, 66,6\% (20) das participantes discordam dessa afirmação. Percebe-se ainda que 13,3\% (04) das participantes acreditam que por fazerem o uso de contraceptivo não acha necessária a realização do exame com frequência, todavia 86,7\% (26) dessas mulheres discordam disso.

Apesar de não ser a maioria, mais de $30 \%$ das mulheres sentem vergonha de realizar o exame o que acaba sendo um empecilho para muitos procurarem o serviço.

Um estudo realizado com 40 mulheres, mostrou que quando questionados acerca dos motivos para não realização do exame de PCCU, metade delas elencaram a vergonha como principal motivo (Dantas, et al., 2018). Em outro estudo com 240 participantes também apontou a vergonha fator determinante para a não realização do exame preventivo (Malta, et al., 2017).

Os dados demonstram ainda um ponto bastante positivo visto que a maioria das mulheres acham necessário a realização do exame de PCCU mesmo fazendo uso de contraceptivo sendo esta uma prática fundamental visto que a contracepção não consiste propriamente em um método para prevenir o câncer do colo do útero. 
Gráfico 3: Confiança e medo em relação ao exame de PCCU.

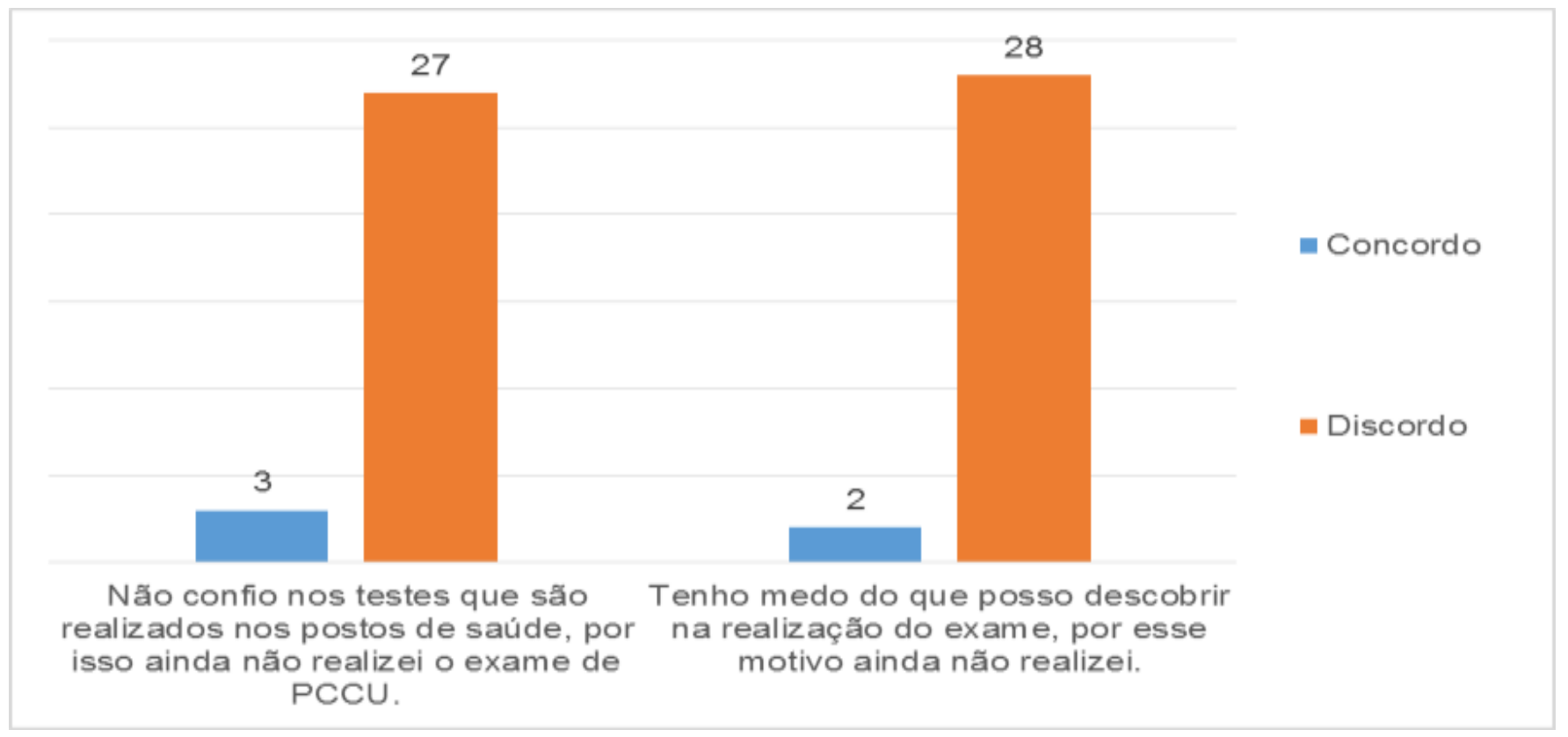

Fonte: Autores (2021).

A partir do Gráfico 3, observa-se que 90\% (27) das participantes confiam nos testes, porém 10\% (03) afirmaram não confiar e elencam esse fato como motivo para a não realização do exame nos postos de saúde. Além disso 6,6\% (02) das participantes dizem ter medo do que podem descobrir durante a realização do exame e por esse motivo não o realizam, todavia observa se que $93,4 \%$ (28) das participantes discordam dessa afirmação.

A literatura demonstra que muitas mulheres ainda se sentem constrangidas e envergonhadas durante a realização do exame demonstrando ainda medo do resultado (Lopes, et al., 2019).

Gráfico 4: Aceitação da parceria e ausência de queixas em relação ao exame de PCCU.

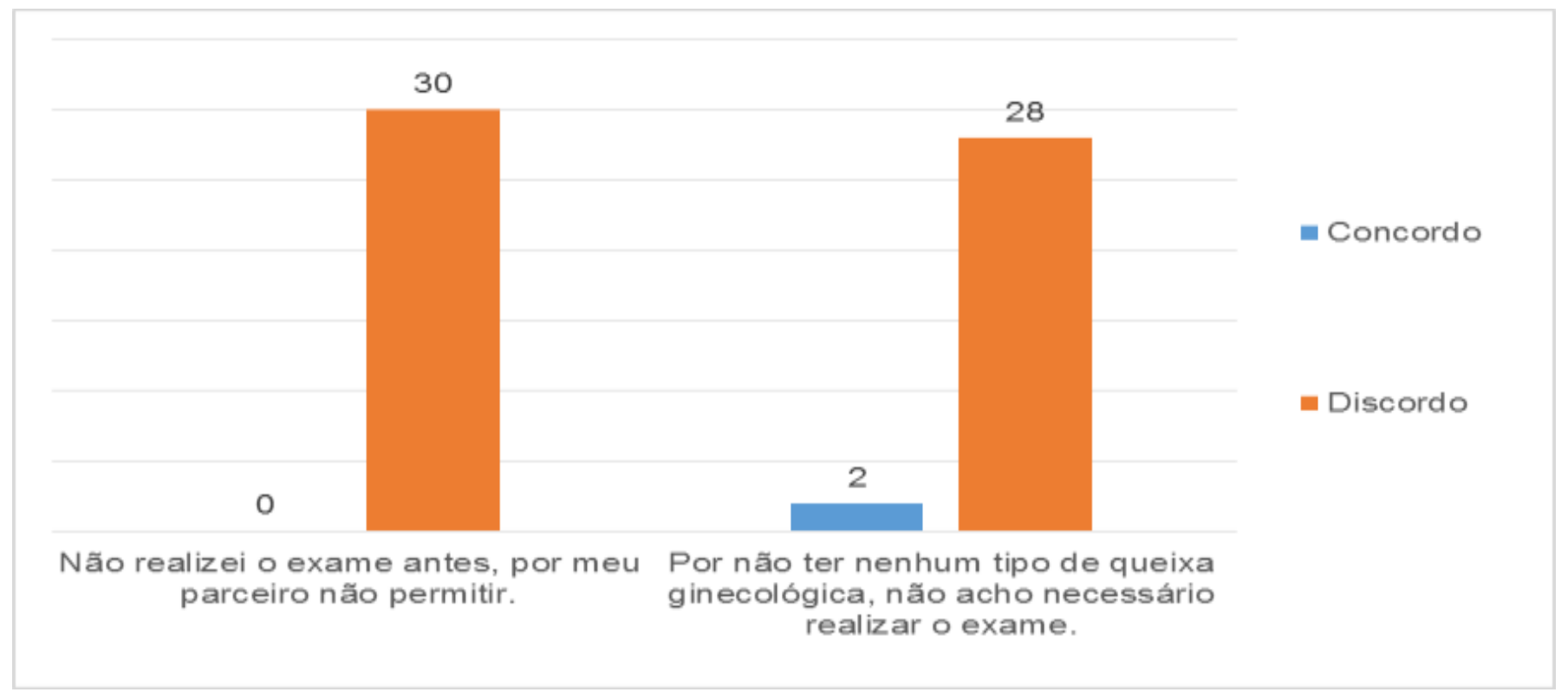

Fonte: Autores (2021). 
O Gráfico 4 demonstra que a permissão do parceiro não influenciou para a não realização do exame em 100\% (30) das participantes, observa-se também que 6,66\% (02) das participantes afirmam que por não terem nenhum tipo de queixa ginecológica acham desnecessário a realização do exame, no entanto, 93,4\% (28) discordam dessa afirmação.

Os dados apresentados são fundamentais para o sucesso das estratégias de promoção à saúde da mulher demonstrando o protagonismo feminino frente ao seu próprio cuidado no ponto em que nenhuma participante da pesquisa condicionou a realização do exame de PCCU a permissão do parceiro além disso, o fato da maioria achar necessário a realização do exame independentemente de queixas encontra-se em consonância com o seu objetivo primordial que trata se da prevenção.

O presente estudo difere de outros da literatura, onde é demonstrado em uma pesquisa realizada em 2019, que a maioria das mulheres que foram realizar o exame de PCCU, o fizeram devido à presença de sinais e sintomas, tendo como predomínio queixas relacionadas a corrimento e prurido vaginal (Silva, et al, 2019).

Tabela 2: Aspectos acerca da realização do exame de PCCU.

\section{PERGUNTAS/RESPOSTAS NÚMEROTOTAL TORCENAGEM}

Qual a frequência para a realização do PCCU?

\begin{tabular}{|c|c|c|}
\hline A cada 6 meses & 3 & $10 \%$ \\
\hline Anualmente & 22 & $73,5 \%$ \\
\hline A cada 2 anos & 1 & $3,3 \%$ \\
\hline A cada 3 anos & 2 & $6,6 \%$ \\
\hline Não souberam responder & 2 & $6,6 \%$ \\
\hline \multicolumn{3}{|c|}{ A partir de qual idade se deve realizar o exame de PCCU? } \\
\hline Após os 18 anos & 4 & $13,3 \%$ \\
\hline Após os 20 anos & 1 & $3,3 \%$ \\
\hline Após os 25 anos & 6 & $20 \%$ \\
\hline Após os 30 anos & 1 & $3,3 \%$ \\
\hline Após os 40 anos & 1 & $3,3 \%$ \\
\hline Após a menstruação & 3 & $10 \%$ \\
\hline Após a primeira relação sexual & 11 & $36,8 \%$ \\
\hline Não souberam responder & 3 & $10 \%$ \\
\hline \multicolumn{3}{|l|}{ Qual o objetivo para a realização do exame de PCCU? } \\
\hline Prevenir o câncer do colo de útero & 15 & $50 \%$ \\
\hline $\begin{array}{l}\text { Identificar e diagnosticar doenças e alterações no } \\
\text { útero }\end{array}$ & 14 & $46,7 \%$ \\
\hline Não souberam responder & 1 & $3,3 \%$ \\
\hline Como o exame é realizado? & & \\
\hline
\end{tabular}




\begin{tabular}{|c|c|c|}
\hline Através de uma coleta do material do útero & 19 & $60,8 \%$ \\
\hline Observação do canal vaginal & 2 & $6,6 \%$ \\
\hline De maneira respeitosa e sigilosa & 5 & $16,6 \%$ \\
\hline Não souberam responder & 4 & $13,3 \%$ \\
\hline
\end{tabular}

Fonte: Autores (2021).

A Tabela 2, apresenta os aspectos acerca da realização do exame de PCCU. Quando questionadas sobre a frequência em que o exame deve ser realizado, a maioria das participantes que corresponde a 73,5\% (02) responderam anualmente, seguido de $10 \%$ (03) que responderam a cada 6 meses, 6,6\% (02) das mulheres afirmaram que a frequência correta dá-se a cada 3 anos, se igualando a esse quantitativo encontra-se as que não souberam responder, por fim 3,3\% (01) responderam a cada 2 anos.

No Brasil a recomendação acerca da periodicidade para realização do exame de PCCU dá-se uma vez ao ano e a partir de dois exames anuais consecutivos negativos sua realização pode ser a cada 3 anos, porém, observa-se que muitas mulheres realizam exame em momentos de procura dos serviços por outros motivos sendo assim um rastreamento oportunístico levando a realização do exame em mulheres de faixa etária fora do recomendado e com intervalo entre eles de um ano ou menos (Brasil, 2016).

A realização periódica do exame consiste em uma estratégia importante eficiente para o rastreamento do câncer do colo de útero tendo em vista que diminui significativamente a incidência e mortalidade pelo câncer em países que apresentam uma cobertura superior a 70\% apresentam taxas iguais ou menores as duas mortes a cada 100 mil mulheres ao ano (Brasil, 2016).

Quanto à pergunta: A partir de qual idade se deve realizar o exame de PCCU? A maioria 36,8 (11) das participantes responderam que deveria ser realizada após a primeira relação sexual, conseguinte 20\% (06) responderam após os 25 anos de idade.

Apesar das inúmeras discussões acerca da definição da população alvo para o exame de PCCU vê-se um consenso que mulheres que nunca tiveram relação sexual não correm o risco para desenvolvimento do câncer de colo do útero. Em mulheres jovens sexualmente ativas podem ser implementadas diferentes medidas de prevenção sem que haja necessidade de inclusão no programa de rastreio devendo ser orientadas quanto à prática do sexo seguro e acerca das infecções sexualmente transmissíveis (IST) (Brasil, 2016).

O Ministério da Saúde orienta que a coleta para exames PCCU deve ser realizado a partir dos 25 anos em mulheres que já iniciaram atividade sexual devendo seguir até os 64 anos de idade e interrompidas após dois exames negativos consecutivos nos últimos cinco anos. A incidência do câncer invasivo do colo do útero em mulheres até 24 anos é baixa no entanto com rastreamento precoce aumentaria o diagnóstico de lesões de baixo grau aumentando assim as possibilidades de regressão (Brasil, 2016).

Em relação à pergunta: Qual o objetivo para realização do exame de PCCU? 50\% (15) referiram que o objetivo consiste em prevenir o câncer de colo do útero, seguido de 46,7\% (14) das participantes que responderam para identificar e diagnosticar doenças e alterações no útero. 
Quando questionadas acerca de como o exame é realizado, 60,8\% (19) responderam ser através de uma coleta de material do colo do útero, 16,6\% (05) disseram ser realizado de maneira respeitosa e sigilosa, 6,6\% (02) atra vés da observação do canal vaginal e $13,3 \%$ (04) não souberam responder.

Quando se fala no exame de PCCU o conhecimento das mulheres é de suma importância visto que a falta deste provoca um prognóstico desfavorável devido a não realização do exame promovendo assim diagnósticos tardios (Gomes, et al., 2017).

Um estudo realizado com 240 mulheres demonstrou que mais de $70 \%$ ouviram falar do exame todavia apenas pouco mais de $14 \%$ o realizaram para prevenir o câncer de colo de útero, onde a maioria das participantes apontaram como a finalidade do exame a detecção de Infecções Sexualmente Transmissíveis (Malta, et al., 2017).

Em relação a finalidade do exame, o estudo de Silva, et al, (2020) mostra que a maioria das mulheres atribuíram a função como a prevenção contra o câncer de colo de útero e em relação ao tempo de realização de um exame ao outro $87 \%$ das mulheres responderam que deveria ser realizado de 6 meses a um ano.

Gomes, et al., (2017), também demonstrou que as mulheres já ouviram falar sobre o exame no entanto desconheciam a sua função bem como sua importância.

Um estudo realizado em 2019 demonstrou que a maioria das participantes apresentavam conhecimento adequado acerca de um exame de PCUU praticavam ações de prevenção ao câncer (Melo, et al., 2019). Em contrapartida a esse resultado Dantas, et al., (2018) evidenciou em seu estudo que o conhecimento das mulheres acerca do exame de PCCU ainda é muito baixo.

Segundo Lopes, et al., (2019), faz-se necessário um olhar holístico do enfermeiro a fim de responder os diferentes questionamentos das mulheres em busca do aumento de adesão ao exame.

\section{Considerações Finais}

A presente pesquisa foi de suma importância para avaliar o conhecimento das mulheres atendidas pela CEPS do IESC/FAG acerca do exame de PCCU, tendo em vista que apesar dos avanços médicos, científicos e tecnológicos ao longo dos anos, o câncer do colo do útero ainda constitui um sério problema de saúde pública.

O estudo demonstrou que as participantes da pesquisa possuem conhecimento regular acerca do exame de PCCU onde reconhecem e compreendem a importância da realização do exame e sua finalidade, estando cientes ainda quanto à periodicidade em que deve ser feito, no entanto, ainda foi possível observar que parte das mulheres entrevistadas desconheciam a idade para iniciar o exame e ainda acerca da maneira de sua realização, relatando ainda vergonha perante o procedimento.

Dessa maneira faz-se necessário a continuidade dos serviços prestados pela referida instituição, onde a enfermagem deve atentar-se cada vez mais para a oportunidade de esclarecimentos de dúvidas no momento da consulta, propiciando uma escuta de excelência que além da realização técnica do exame engloba todos os seus aspectos, levando ainda mais conhecimento para as mulheres visto que este é um fator fundamental para a adesão ao exame, sendo possível assim diminuir o número do câncer de colo do útero.

\section{Referências}

Acosta, D. F. et al. (2017). Vivenciando o exame Papanicolau: entre o (não) querer e o fazer. Revista de Enfermagem UFPE on line, [S.1.], 11(8), 3031-3038, mar. 2017. ISSN 1981-8963.

Amaral, M.S.; Gonçalves, A.G.; \& Silveira, L.C.G. (2017). Prevenção do câncer de colo de útero: a atuação do profissional enfermeiro nas unidades básicas de saúde. Revista Científica FacMais, Volume. VIII, Número 1. Fev/Mar. Ano 2017/1 Semestre. ISSN $2238-8427$. 
Brasil. (2016). Instituto Nacional de Câncer José Alencar Gomes da Silva. Coordenação de Prevenção e Vigilância. Divisão de Detecção Precoce e Apoio à Organização de Rede. Diretrizes brasileiras para o rastreamento do câncer do colo do útero / Instituto Nacional de Câncer José Alencar Gomes da Silva. Coordenação de Prevenção e Vigilância. Divisão de Detecção Precoce e Apoio à Organização de Rede. - (2. ed. rev. atual.) - Rio de Janeiro: INCA, 2016.

Brasil. (2020). Instituto Nacional de Câncer José Alencar Gomes da Silva (INCA). Estimativa 2020: incidência do Câncer no Brasil. Rio de Janeiro: INCA 2019.

Brasil. (2021). Instituto Nacional de Câncer José Alencar Gomes da Silva (INCA). Atlas da mortalidade. Rio de Janeiro: INCA, 2021.

Carvalho, R.S. et al. (2016). Perfil preventivo do câncer de colo uterino em trabalhadoras da enfermagem. Revista de Enfermagem UFPE on line, [S.1.], 11(6), 2257-2263, dez. 2016. ISSN 1981-8963

Ceolin, R. et al. (2020). Analysis of cancer trace cancer of the uteruscolumn of a municipality of the south of brazil / Análise do rastreamento do câncer do colo do útero de um município do sul do Brasil. Revista de Pesquisa: Cuidado é Fundamental Online, [S.1.], 12, 406-412, july 2020. ISSN 2175-5361.

Dantas, P.V. J. et al. (2018). Conhecimento das mulheres e fatores da não adesão acerca do exame papanicolau. Rev enferm UFPE on line. Recife, 12(3):68491, mar., 2018.

Fernandes, N.F.S. et al. (2019). Acesso ao exame citológico do colo do útero em região de saúde: mulheres invisíveis e corpos vulneráveis. Cad. Saúde Pública, Rio de Janeiro, 35(10), e00234618, 2019.

Gasparin, V. A. et al. (2020). Rastreamento do câncer de colo do útero durante o acompanhamento pré-natal. Revista Eletrônica De Enfermagem, 22.

GOMES, L. C. de S. et al. (2017). Conhecimento de mulheres sobre a prevenção do câncer de colo do útero: uma revisão integrativa. Revista Uningá Review, [S.1.], 30(2), maio 2017. ISSN 2178-2571.

Iarc. (2020). INTERNATIONAL AGENCY FOR RESEARCH ON CANCER (IARC). Cancer today. Lyon: WHO, 2020.

Lopes, J.C. et al. (2019). O Papel do Enfermeiro no conhecimento das Mulheres acerca do Exame de Papanicolau / The Role of the Nurse in the knowledge of Women about Papanicolau Examination. ID on line. Revista de psicologia, [S.1.], 13(47), 527-537, out. 2019. ISSN 1981-1179.

Malta, E.F.G.D. et al. (2017). Prática inadequada de mulheres acerca do papanicolaou. Texto \& Contexto - Enfermagem. 2017 26(1), e5050015.

Martins, P.C.A.; \& Motta, I.S. da. (2020). O saber acadêmico sobre as competências do enfermeiro na prevenção do câncer do colo do útero. Enferm Bras 2020;19(1):4-10 https://doi.org/10.33233/eb.v19i1.3977.

Melo, E.M.F. de, et al. (2019). Câncer de colo uterino: conhecimento, atitude e prática sobre o exame de prevenção. Rev. Bras. Enferm. 2019, 72(3), 25-31. Epub 13-Dez-2019. ISSN 1984-0446. http://dx.doi.org/10.1590/0034-7167-2017-0645.

Silva, J. N. da; et al. (2020). Exame de papanicolaou: conhecimentos de mulheres em uma unidade de saúde escola da Amazônia ocidental. Revista Eletrônica Acervo Saúde, 12(7), e3312, 21 maio 2020.

Silva, A. B. et al. (2019). Adesão das mulheres ao exame citopatológico para prevenção do câncer cervicouterino. Revista Ciência Plural, 4(3), 69-81, 4 abr. 2019.

Silva, R.L. da, et al. (2019). A importância da medicina preventiva na prevenção e diagnóstico do câncer de colo útero nas mulheres em idade fértil na estratégia saúde da família- Tenoné II no município de Belém-PA. Universidade Federal do Pará. Universidade Aberta do SUS. Curso de Especialização em Saúde da Família. BELÉM - PA 2019.

Silva, J. F. T. et al. (2021). A percepção das mulheres quanto à prevenção do câncer do colo do útero e a realização do exame de Papanicolaou. Pesquisa, Sociedade e Desenvolvimento, [S. eu.], 10(22), e368101220525, 2021. DOI: 10.33448 / rsd-v10i12.20525.

Teixeira, V. R. S. et al. (2018). A Segurança do Paciente diante da Assistência de Enfermagem na coleta do exame Papanicolau em uma Estratégia Saúde da Família: um relato de experiência. Revista Eletrônica Acervo Saúde, 11(3), e205, 29 dez. 2018.

Vasconcelos, L. C. et al. (2017). Conhecimento de Mulheres a Respeito do Exame Papanicolau. Uniciências, 21(2), 105-109, 2017. 